\title{
Charge reconfiguration in arrays of quantum dots
}

\author{
Johannes C. Bayer, ${ }^{*}$ Timo Wagner, Eddy P. Rugeramigabo, and Rolf J. Haug \\ Institut für Festkörperphysik, Leibniz Universität Hannover, 30167 Hannover, Germany \\ (Received 8 May 2017; revised manuscript received 9 November 2017; published 15 December 2017)
}

\begin{abstract}
Semiconductor quantum dots are potential building blocks for scalable qubit architectures. Efficient control over the exchange interaction and the possibility of coherently manipulating electron states are essential ingredients towards this goal. We studied experimentally the shuttling of electrons trapped in serial quantum dot arrays isolated from the reservoirs. The isolation hereby enables a high degree of control over the tunnel couplings between the quantum dots, while electrons can be transferred through the array by gate voltage variations. Model calculations are compared with our experimental results for double, triple, and quadruple quantum dot arrays. We are able to identify all transitions observed in our experiments, including cotunneling transitions between distant quantum dots. The shuttling of individual electrons between quantum dots along chosen paths is demonstrated.
\end{abstract}

DOI: 10.1103/PhysRevB.96.235305

\section{INTRODUCTION}

Arrays of quantum dots (QDs) are expected to be important tools for studying electronic properties of quantum mechanical circuits. Electrons confined in QDs can be controlled and manipulated, allowing implementation as spin [1-3] and charge [4-6] qubit devices. The potential scalability of semiconductor based QD qubits has already been demonstrated by the implementation of triple [7,8], quadruple [9-11], and recently by the realization of a quintuple quantum dot [12]. While larger QD arrays provide more functionality compared to single or double QD spin qubits [13] the number of control parameters and therefore tuning complexity increases significantly. It is thus crucial to find reproducible, noncomplex, and scalable methods for tuning QD arrays in a defined way. Isolating the QDs from the electronic reservoirs has already been shown to significantly simplify the stability diagram of double quantum dots $[14,15]$ while enabling the possibility to control the exchange interaction between two electron spins by tuning the tunnel coupling [16] instead of detuning the dot potentials. Here we demonstrate the isolation of QD arrays with up to four QDs and discuss the arising phenomena.

\section{EXPERIMENT AND SIMULATION}

Our quantum dot device is based on a GaAs/AlGaAs heterostructure, forming a two-dimensional electron gas (2DEG) with an electron density of $n_{s}=2.4 \times 10^{11} \mathrm{~cm}^{-2}$ and mobility of $\mu=5.1 \times 10^{5} \mathrm{~cm}^{2} /(\mathrm{Vs})$ at approximately $100 \mathrm{~nm}$ below the surface. A scanning electron microscope (SEM) image of a device similar to the one used is shown in Fig. 1. The quadruple quantum dot (QQD) device consists of a series of metallic Schottky gates: five tunnel barrier gates Tgi, four plunger gates Dgi, and two bottom gates Bgi. Quantum dot $i$ is thus defined by the two tunnel barrier gates $\operatorname{Tg} i$ and $\operatorname{Tg}(i+1)$, the plunger gate $\mathrm{Dg} i$, and one or both of the larger gates $\mathrm{Bg} 1$ and $\mathrm{Bg} 2$. Interdot tunnel couplings between neighboring QDs as well as dot-reservoir couplings can be manipulated via Tgs, while energy levels can be controlled via Dgs. Two additional gates are attached below the gates $\mathrm{Bg} 1$ and $\mathrm{Bg} 2$, which form

\footnotetext{
*Corresponding author: bayer@nano.uni-hannover.de
}

quantum point contacts (QPCs), used as charge detectors sensitive to all four QDs. For all measurements shown, only the right charge detector Qg1 is used. The gap between gates $\mathrm{Bg} 1$ and $\mathrm{Bg} 2$ is electrostatically closed by applying sufficiently negative potentials of $V_{B g 1}=V_{B g 2}=-0.4 \mathrm{~V}$. All measurements were performed in a dilution refrigerator at a base temperature of $10 \mathrm{mK}$ and with a measurement frequency of $28.6 \mathrm{~Hz}$.

By applying negative potentials to the respective gates, we are able to tune in all desired configurations from single to quadruple quantum dots. This allows the investigation of electron shuttling in serial QD arrays beginning with the well understood double quantum dot and extending the system up to the quadruple quantum dot. Capacitive model simulations were performed to complement our understanding of the charge reconfigurations. The capacitive model includes up to four QDs and two gates, capacitances between gates and QDs, mutual capacitances between neighboring QDs, and yields the electron numbers occupying the QDs. The influence of long range transitions between distant QDs can be illustrated by enabling or disabling the respective transitions. In the following, these long range transitions will be referred to as cotunneling transitions.

\section{RESULTS AND DISCUSSIONS}

\section{A. Double quantum dot}

A double quantum dot (DQD) charge stability diagram of QDs 2 and 3 is shown in Fig. 2(a) as a function of the outer tunnel barrier gate voltages $V_{T g 2}$ and $V_{T g 4}$ with $V_{T g 2}$ being swept from more positive to more negative values. The stability diagram can be divided into three parts, which differ in coupling between QDs and reservoirs. Above the horizontal line the DQD is coupled to the right reservoir. On the right side of the vertical line, the DQD is coupled to the left reservoir. Consequently, we are observing the DQD typical honeycomb pattern [17] in region I. In both regions II, the DQD is decoupled from one reservoir. Here the honeycomb pattern is only preserved if cotunneling mediated transitions are allowed. If cotunneling is suppressed, the charging lines of the dot which is not directly coupled to the reservoir change into staircaselike 


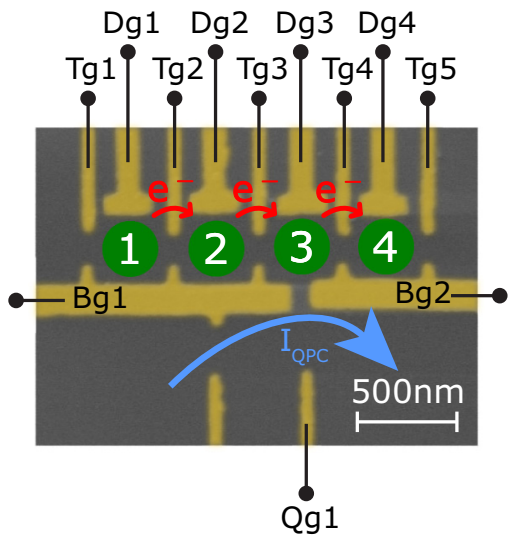

FIG. 1. False color SEM image of the QQD device; the QDs are depicted as green circles. All measurements shown are performed using the QPC charge detector defined by Qg1. As indicated by the red arrows, electrons can be shifted through the QD system by gate voltage variations.

shape [18], since charges can only be exchanged with the other dot.

In region III the DQD becomes fully isolated with a fixed number of electrons trapped [14-16]. The resulting pattern in the detector signal originates only from charge reconfigurations inside the isolated DQD system. For the given sweep direction, charges are exclusively transferred from QD2 to QD3. The electrons are thus shifted towards the detector which leads to a decrease in detector current (dark blue). If an electron is removed from the vicinity of the detector, e.g., for electrons tunneling from QD2 into a reservoir, the detector current increases (white/red). By counting the number of charge reconfiguration lines occurring in one sweep we can thus determine the number of electrons that populated QD2 at the isolation point. Capacitive model simulations were performed using energy level alignment and capacitance ratios extracted from the measurement. To be equivalent to the experimental detector signal, the electron numbers are linearly combined and derived in $V_{T g 2}$ direction. The resulting graph is shown in Fig. 2(b). Isolation threshold values $V_{t 1}$ (vertical red line) and $V_{t 2}$ (horizontal red line) are defined, which allows partitioning the outcome in regions I, II, and III, similar to the experiment. Our purely electrostatic simulation is naturally not able to reproduce the tunnelcoupling induced broadening of the reconfiguration lines; the general reconfiguration pattern (region III), however, is in good agreement with our experimental observations.

The large increase in spacing from the charging lines of QD2 in the open region $V_{T g 2}>-0.7 \mathrm{~V}$ (vertical red line) to the charge reconfiguration lines in the isolated system $\left(V_{T g 2}<-0.7 \mathrm{~V}\right)$ can be explained by a combination of two effects. Resonances between QD2 and the reservoir occur in distances proportional to the capacitance between $\mathrm{Tg} 2$ and QD2, whereas resonances between QD2 and QD3 occur only proportional to the relative energetic shift of the QD levels. Figures 2(d)-2(f) show energy diagrams of three different resonance conditions for the given electron configuration $\left(n_{2}, n_{3}\right)=(2,0)$ at the isolation point. These situations are also marked in Figs 2(a) and 2(b). At the first resonance, denoted
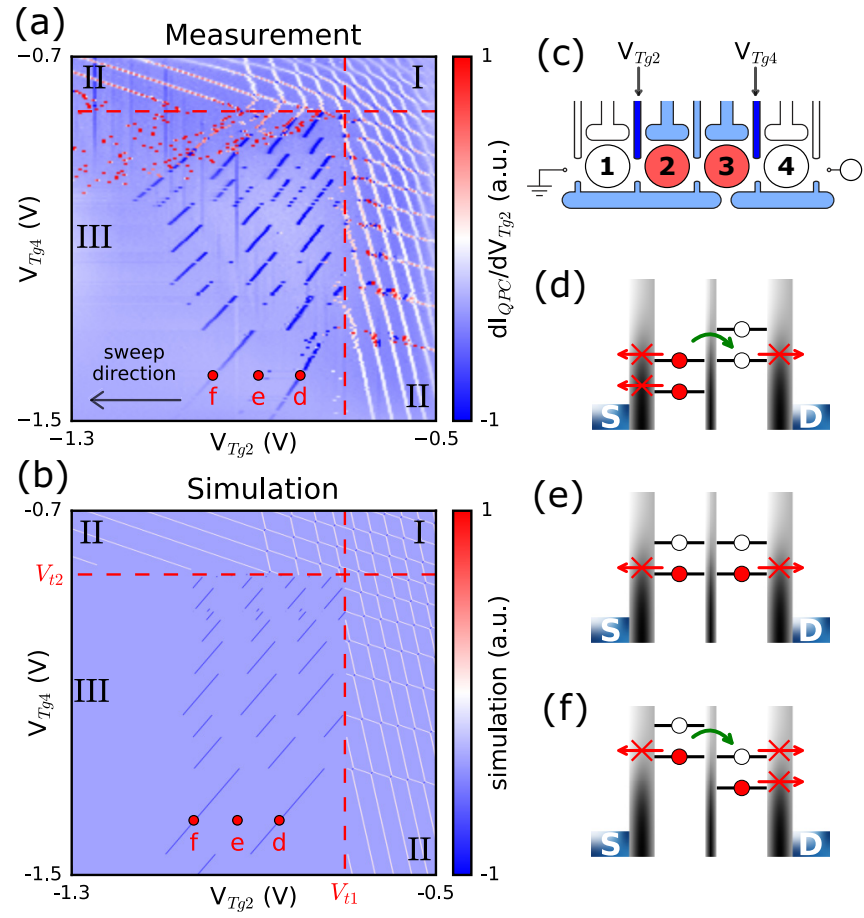

(e)

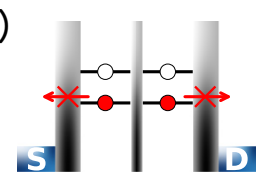

(f)

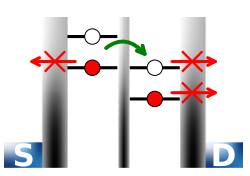

FIG. 2. (a) DQD stability diagram as a function of $V_{T g 2}$ and $V_{T g 4}$. Red dashed lines represent the isolation threshold, where tunneling rates through the respective barrier drop below the measurement frequency. (b) Calculated capacitive model stability diagram. (c) Scheme of the DQD setup. Light blue gates are at fixed voltages, dark blue gates are varied for the measurement, and white gates are grounded. Only the reddish colored DQD is defined. (d)-(f) Energy level schemes of the three points marked in (a) and (b). Panel (d) marks the $(2,0) \rightarrow(1,1)$ transition, (e) the resonance between the lowest level of both QDs, and (f) the $(1,1) \rightarrow(0,2)$ transition.

by (d), the second electron of QD2 is transferred to QD3: $(2,0) \rightarrow(1,1)$. The next resonance when moving towards more negative $V_{T g 2}$ is between the first level of both QDs (e). No transition occurs, since both resonant energy levels are already occupied. At the third resonance (f), the remaining electron on QD2 is transferred to QD3: $(1,1) \rightarrow(0,2)$. The fact that only every second resonance appears as a transition is yet another proof that electrons are trapped inside the DQD system. Adjusting the plunger gate voltages allows controlling the number and distribution of electrons trapped in the DQD system when isolating the system.

\section{B. Triple quantum dot}

This concept of isolating and manipulating a well defined number of electrons in a serial multi-QD becomes even more relevant when scaling up the system. While the stability diagram of a triple quantum dot (TQD) system coupled to the reservoirs is three dimensional [8], the number of independent energy scales in an isolated TQD stability diagram is reduced to two. A stability diagram of an isolated TQD as a function of the outer plunger gate voltages $V_{D g 1}$ and $V_{D g 3}$ is shown in Fig. 3(a). The use of plunger gates allows the investigation of a larger region in the TQDs energy space. 
(a)

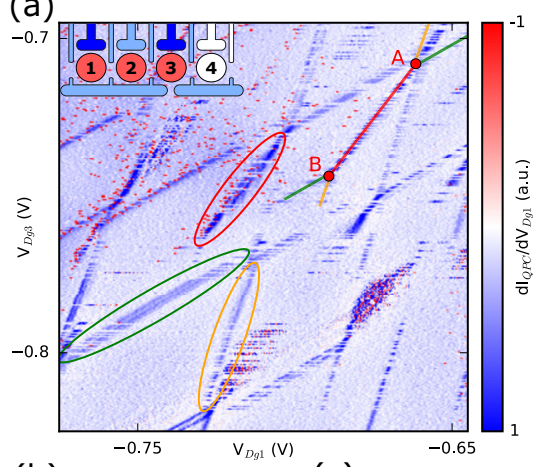

(b)
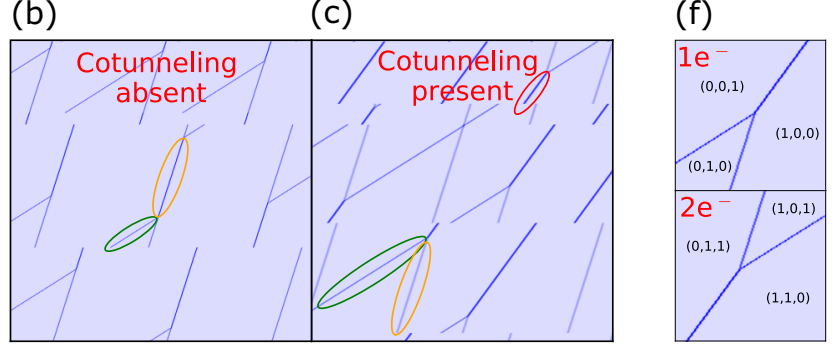

FIG. 3. (a) Isolated TQD stability diagram as a function of $V_{D g 1}$ and $V_{D g 3}$ with inset sample scheme similar to Fig. 2. Sequential transitions are marked in green/orange; the cotunneling transition $(1 \rightarrow 3)$ is marked in red. Points A and B mark two TQD resonances occurring for identical charge configurations at the isolation point. (b) Capacitive model simulations excluding and (c) including transitions directly from QD1 to QD3. Transitions marked as in (a). (d) Scheme of cotunneling transitions directly between both outer QDs via two different virtual states of the detuned center QD. (e) Scheme of a TQD resonance in the isolated region. (f) Zoom into simulated resonances, allowing the distinction between resonances occupied by one (top) or two electrons (bottom).

As in the DQD system, the system was reset to a state well coupled to at least one reservoir at the beginning of each line, so the number of electrons trapped in the TQD is constant in the $V_{D g 1}$ direction, while it may change in the $V_{D g 3}$ direction. Three different slopes of charge reconfiguration lines are observed in the isolated TQD stability diagram. A reconfiguration line, corresponding to an electron being transferred from QD1 to QD2, is marked by an orange ellipse in Fig. 3(a). The slope highlighted by the green ellipse corresponds to electrons being transferred from QD2 to QD3. The transitions leading to the intermediate slope, highlighted by a red ellipse, can be derived by comparing two capacitive model simulations. Figure 3(b) shows a stability diagram of a TQD in the isolated regime with only nearest neighbor transitions allowed. Only two different slopes of reconfiguration lines are observed (orange, green). By allowing transitions directly from QD1 to QD3, the outcome changes to what is shown in Fig. 3(c). A third slope of reconfiguration lines develops, consistent with our experimental outcome. These reconfiguration lines occur for resonances between QD1 and QD3 with QD2 being off-resonant, which can be understood by taking higher order coherent tunneling, e.g., cotunneling via virtual states of the center QD, into account [19].

Whenever two of the reconfiguration lines meet in the isolated TQD case, all three QDs are in resonance and thus three charge configurations are degenerate [Fig. 3(e)]. Comparable to open DQD systems, two species of resonances are emerging [20]. These are resonances with degenerate charge states $(1,0,0),(0,1,0)$, and $(0,0,1)$, and the other possibility with a degeneracy between the $(1,1,0),(1,0,1)$, and $(0,1,1)$ states, where zero refers to the background charge configuration. These two cases can be distinguished by comparing the reconfiguration lines surrounding the resonances. Figure 3(f) shows two zooms in different resonances of the simulation for Fig. 3(c), where cotunneling is present. The first case (top), where only one electron occupies the resonance, cotunneling from QD 1 to 3 is the energetically favorable transition at more positive $V_{D g 3}$ with respect to the resonance, while sequential tunneling is energetically favorable at more negative $V_{D g 3}$. For the second type (bottom), where two electrons occupy the resonance, sequential transitions are observed at more positive $V_{D g 3}$, while cotunneling is more favorable at more negative $V_{D g 3}$.

Points $\mathrm{A}$ and $\mathrm{B}$ in Fig. 3(a) mark two resonances for identical electron configurations at the isolation point and the connected lines highlight all transitions relevant for these resonances. Resonance A is occupied by two electrons and resonance $\mathrm{B}$ by only one electron. Both resonances are connected by a cotunneling reconfiguration line (red). Along this line, the detuning of the center QD2 with respect to the resonant states changes by exactly the charging energy $E_{C}$ of the center QD2. Therefore, two different virtual states are contributing to this reconfiguration line. The virtual transition via the occupied state $N$ [Fig. 3(d), lower path] of QD2, detuned by $\Delta E_{N}$ with respect to the resonant levels of QD1 and QD3, and via the unoccupied state $N+1$ [Fig. 3(d), upper path] with detuning $\Delta E_{N+1}$. Beginning at resonance $\mathrm{A}$, the detunings are $\Delta E_{N}=0$ and $\Delta E_{N+1}=E_{C}$. In the center of the cotunneling reconfiguration line $\Delta E_{N}=\Delta E_{N+1}=E_{C} / 2$ and at resonance $\mathrm{B}$ the detunings are given by $\Delta E_{N}=E_{C}$ and $\Delta E_{N+1}=0$, with $E_{C} \approx 1.4 \mathrm{meV}$. This means the detunings and therefore the weight of these two cotunneling processes can be modulated by moving along this reconfiguration line. Additionally, the isolation enables efficient tunability of the interdot tunnel couplings $\Gamma_{12}$ between QD1 and QD2 and $\Gamma_{23}$ between QD2 and QD3 by changing the tunnel barrier gate voltages. This enables a way to control the exchange interaction [16] for long range transitions in isolated QD arrays. For the given case, the tunnel couplings were extracted from the broadening of the sequential reconfiguration lines and are $\Gamma_{12}=74 \pm 7 \mu \mathrm{eV}$ and $\Gamma_{23}=73 \pm 10 \mu \mathrm{eV}$.

\section{Quadruple quantum dot}

By tuning in the fourth QD, the same kind of isolation can be achieved on a quadruple quantum dot (QQD) system. A stability diagram of an isolated QQD system as a function of the plunger gate voltages $V_{D g 1}$ and $V_{D g 4}$ is shown in Fig. 4(a). When considering all cotunneling transitions of the QQD system, there are six different possible transitions: three sequential ones, $(1 \rightarrow 2),(2 \rightarrow 3)$, and $(3 \rightarrow 4)$, two next nearest neighbor cotunneling transitions $(1 \rightarrow 3)$ and $(2 \rightarrow 4)$, as well as a cotunneling transition directly from the leftmost to the rightmost QD $(1 \rightarrow 4)$. 

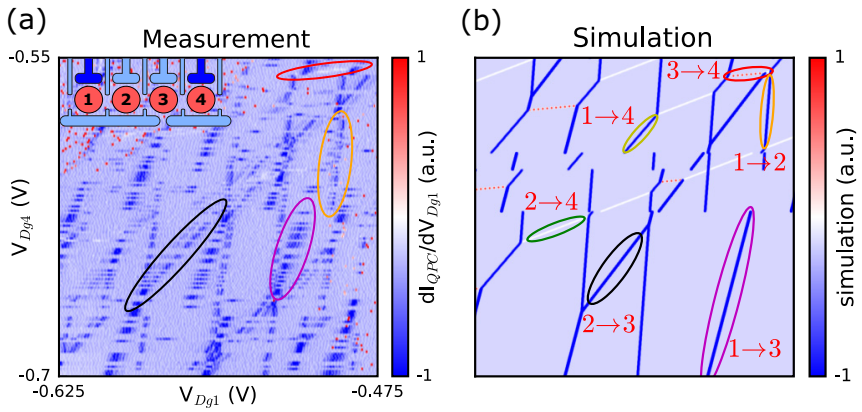

FIG. 4. (a) Isolated QQD stability diagram as a function of $V_{D g 1}$ and $V_{D g 4}$ with inset sample scheme. Transitions are marked according to the simulation. (b) Capacitive model simulation of an isolated QQD stability diagram. One of each of the six possible transitions is marked by an ellipse.

In our QQD simulation, shown in Fig. 4(b), the linear combination of electron numbers was chosen in a way that all six transitions can be clearly identified. White and red colored lines hereby appear for charges shifted away from the detector, e.g., for transitions from QD3 to QD4. The capacitance values used for the simulations are extracted from the measurements, making the reconfiguration line slopes directly comparable with our experimental data. By this, we are able to identify four of the transitions and the corresponding slopes of the reconfiguration lines in the isolated QQD stability diagram in Fig. 4(a). The highest slope, marked by the orange ellipse, can be identified as the transition $(1 \rightarrow 2)$. Cotunneling transitions $(1 \rightarrow 3)$ experience a stronger impact of $V_{D g 4}$ and one is marked by the magenta colored ellipse. The almost diagonal slope, marked in black, corresponds to transitions $(2 \rightarrow 3)$ and the almost horizontal slope (red) belongs to $(3 \rightarrow 4)$ transitions which appear as white lines since charge is shifted away from the detector. The two remaining transitions $(2 \rightarrow 4)$ and $(1 \rightarrow 4)$, green and yellow ellipse in Fig. 4(b), however, cannot be clearly identified in our experimental data. For the $(2 \rightarrow 4)$ transition, this might be due to the QDs being arranged symmetrically around the detector, which leads to a vanishing current change. For the $(1 \rightarrow 4)$ cotunneling transition the tunneling probability via two QDs is expected to be low. Additionally, the transition is almost similarly affected by both gate voltages $V_{D g 1}$ and $V_{D g 4}$ and the expected reconfiguration line slope is thus again close to diagonal. Cotunneling transitions have already been intensively studied as a limiting factor for the accuracy of junction electron pumps [21-23]. Theoretical calculations of cotunneling rates [24-26] show that, despite the long distance, even the $(1 \rightarrow 4)$ transition exists. The efficient control over the tunnel barriers in isolated systems should therefore allow the tuning of these transitions. The general existence of cotunneling transitions however opens up the counterintuitive possibility of shuttling electrons circularly through a linear array of quantum dots. The experimentally well pronounced $(1 \rightarrow 3)$ transition for example allows the implementation of $(1 \rightarrow 3 \rightarrow 2 \rightarrow 1)$ electron loops. The equivalent $(2 \rightarrow 4 \rightarrow$ $3 \rightarrow 2$ ) loop should be similarly tunable. The loop resulting from the $(1 \rightarrow 4)$ transition would be $(1 \rightarrow 4 \rightarrow 3 \rightarrow 2 \rightarrow$ 1). All combinations of these loops can be used to create complex electron paths through the linear array of QDs.

When comparing the DQD, TQD, and the QQD in the isolated regime, the experimentally observed reconfiguration lines appear more and more fragmented with increasing number of QDs. This can be explained by fluctuations in the charge configuration at the point where the system becomes isolated. In all presented measurements, the isolation pattern is observed by decoupling the right reservoir and sweeping a gate, which decouples the left reservoir as well. The longer the array of quantum dots, the weaker the coupling from the right QD to the left reservoir gets. Low coupling strengths, however, lead to metastable states even before the point of isolation is reached. The electron configuration at the isolation point thus depends on statistical tunneling events and the lifetimes of the arising metastable states compared to our measurement frequency. Possibilities to overcome this would lie in the introduction of waiting times at the isolation point or a pulse sequence opening the right barrier for a certain time interval.

Tuning a linear device of coupled QDs into the regime where every QD is charged with only one electron is a key issue for the implementation of QD based qubit architectures [11]. The scheme of isolated QD arrays opens up a scalable method for reaching this regime while profiting from the possibility of manipulating the interdot tunnel couplings over orders of magnitude without detuning the energy levels [16]. Beginning with a single isolating barrier, the QDs can be tuned into the zero electron regime one after another up to the last QD of the array, which is loaded by a certain amount of electrons and then decoupled from the reservoir. The trapped electrons can now freely be redistributed into any configuration.

\section{CONCLUSION}

To conclude, we investigated here the redistribution of electrons trapped in serial quantum dot arrays decoupled from the reservoirs. Simulations nicely reproduce our experimental observations and enable identification of all transitions occurring in isolated double, triple, and quadruple quantum dots, including cotunneling transitions between distant quantum dots.

\section{ACKNOWLEDGMENTS}

We acknowledge financial support from the School for Contacts in Nanosystems and the Hannover School for Nanotechnology and thank F. Gallego-Marcos and G. Platero for fruitful discussions.
[1] J. R. Petta, A. C. Johnson, J. M. Taylor, E. A. Laird, A. Yacoby, M. D. Lukin, C. M. Marcus, M. P. Hanson, and A. C. Gossard, Science 309, 2180 (2005).
[2] F. H. L. Koppens, C. Buizert, K. J. Tielrooij, I. T. Vink, K. C. Nowack, T. Meunier, L. P. Kouwenhoven, and L. M. K. Vandersypen, Nature (London) 442, 766 (2006). 
[3] R. Hanson, L. P. Kouwenhoven, J. R. Petta, S. Tarucha, and L. M. K. Vandersypen, Rev. Mod. Phys. 79, 1217 (2007).

[4] K. D. Petersson, J. R. Petta, H. Lu, and A. C. Gossard, Phys. Rev. Lett. 105, 246804 (2010).

[5] D. Kim et al., Nat. Nanotechnol. 10, 243 (2015).

[6] A. Stockklauser, P. Scarlino, J. V. Koski, S. Gasparinetti, C. K. Andersen, C. Reichl, W. Wegscheider, T. Ihn, K. Ensslin, and A. Wallraff, Phys. Rev. X 7, 011030 (2017).

[7] D. Schröer, A. D. Greentree, L. Gaudreau, K. Eberl, L. C. L. Hollenberg, J. P. Kotthaus, and S. Ludwig, Phys. Rev. B 76, 075306 (2007).

[8] M. C. Rogge and R. J. Haug, New J. Phys. 11, 113037 (2009).

[9] R. Thalineau, S. Hermelin, A. D. Wieck, C. Bäuerle, L. Saminadayar, and T. Meunier, Appl. Phys. Lett. 101, 103102 (2012).

[10] M. R. Delbecq, T. Nakajima, T. Otsuka, S. Amaha, J. D. Watson, M. J. Manfra, and S. Tarucha, Appl. Phys. Lett. 104, 183111 (2014).

[11] A. Bogan, L. Bergeron, A. Kam, P. Zawadzki, S. Studenikin, L. Gaudreau, and A. Sachrajda, Appl. Phys. Lett. 109, 173108 (2016).

[12] T. Ito et al., Sci. Rep. 6, 39113 (2016).

[13] L. Gaudreau, G. Granger, A. Kam, G. C. Aers, S. A. Studenikin, P. Zawadzki, M. Pioro-Ladrière, R. Wasilewski, and A. S. Sachrajda, Nat. Phys. 8, 54 (2011).
[14] A. W. Rushforth, C. G. Smith, M. D. Godfrey, H. E. Beere, D. A. Ritchie, and M. Pepper, Phys. Rev. B 69, 113309 (2004).

[15] A. C. Johnson, C. M. Marcus, M. P. Hanson, and A. C. Gossard, Phys. Rev. B 71, 115333 (2005).

[16] B. Bertrand, H. Flentje, S. Takada, M. Yamamoto, S. Tarucha, A. Ludwig, A. D. Wieck, C. Bäuerle, and T. Meunier, Phys. Rev. Lett. 115, 096801 (2015).

[17] W. Van der Wiel, S. De Franceschi, J. M. Elzerman, T. Fujisawa, S. Tarucha, and L. P. Kouwenhoven, Rev. Mod. Phys. 75, 1 (2002).

[18] C. H. Yang, A. Rossi, N. S. Lai, R. Leon, W. H. Lim, and A. S. Dzurak, Appl. Phys. Lett. 105, 183505 (2014).

[19] M. Busl, G. Granger, L. Gaudreau, R. Sánchez, A. Kam, M. Pioro-Ladrière, S. A. Studenikin, P. Zawadzki, Z. R. Wasilewski, A. S. Sachrajda, and G. Platero, Nat. Nanotechnol. 8, 261 (2013).

[20] R. H. Blick, R. J. Haug, J. Weis, D. Pfannkuche, K. V. Klitzing, and K. Eberl, Phys. Rev. B 53, 7899 (1996).

[21] J. M. Martinis, M. Nahum, and H. D. Jensen, Phys. Rev. Lett. 72, 904 (1994).

[22] M. W. Keller, J. M. Martinis, N. M. Zimmerman, and A. H. Steinbach, Appl. Phys. Lett. 69, 1804 (1996).

[23] M. W. Keller, A. L. Eichenberger, J. M. Martinis, and N. M. Zimmerman, Science 285, 1706 (1999).

[24] H. D. Jensen and J. M. Martinis, Phys. Rev. B 46, 13407 (1992).

[25] V. I. Mel'nikov, Phys. Lett. A 176, 267 (1993).

[26] P. Lafarge and D. Esteve, Phys. Rev. B 48, 14309 (1993). 